\title{
Realidade Aumentada na Visualização de Soluções do Projeto de Arquitetura
}

\author{
Augmented Reality in the Viewing of Architectural Design Solutions \\ - Lorena Claudia de Souza Moreira \\ UNICAMP, Brasil \\ lorena_moreira@hotmail.com \\ - Regina Coeli Ruschel \\ UNICAMP, Brasil \\ ruschel@fec.unicamp.br
}

\begin{abstract}
Architecture is an area of study in which design solution visualization is essential. It is during the design phase that the projective doubts are clarified. Augmented Reality $(A R)$ enables the visualization of virtual elements in the real environment. In this paper, visualization strategies will be presented in Augmented Reality for the discussion of alternatives design solution in architecture. These strategies are presented in an experiment in which perceptions of users are observed through a workshop. We compare two display strategies (AR mobile $\mathrm{x} A R$ projected) in four types of representation. As a contribution, we present a critical analysis of the benefits and limitations of the strategies used.
\end{abstract}

Keywords: Augmented Reality, Architecture, Design Visualization, Virtual Reality

\section{Introdução}

A Realidade Virtual(RV) ea Realidade Aumentada (RA) podem ser consideradas tecnologias de relevância para arquitetos e urbanistas no suporte a visualização e comercialização do produto final gerado (AGUIAR, 2012). A RV implementa interfaces em três dimensões, proporcionando manipulação e visualização semelhante ao mundo real por meio de artefatos tecnológicos. A RA permite a complementação da visualização do ambiente real com a sobreposição ou combinação de objetos virtuais (AZUMA, 1997).

A Realidade Aumentada é multidisciplinar e sua aplicação tem crescido nos últimos anos, pode-se destacar a visualização de elementos construtivos, sinalização de ambientes e inserção de informações nas áreas da Arquitetura, Engenharia e Construção (AEC). Com a tecnologia cada vez mais robusta e acessível, a criação de aplicações em Realidade Aumentada tem se tornado viável no acréscimo de poder visual de profissionais e clientes, antecipando eventos e otimizando processos, com tendência a participação em toda a cadeia de desenvolvimento de um edifício (MOREIRA, 2013). Constatou-se a viabilidade da aplicação de RA no projeto participativo durante o processo de concepção do projeto arquitetônico (CUPERSCHMID, 2014) e como ferramenta de aprendizagem nos cursos de engenharia estão sendo desenvolvidos experimentos constatando a viabilidade da aplicação (SHIRAZI; BEHZADAN, 2014). Entretanto, estudo envolvendo a incorporação da RA em avaliação pós-ocupação aponta para limitações que ainda inviabilizam a solução tecnológica validada (FREITAS; RUSCHEL, 2015). Dessa forma, confirma-se o diagnóstico de Wang et al. (2013), que afirma que os sistemas de RA aplicados ao ambiente construído ainda se apresentam como protótipos laboratoriais requerendo uma validação industrial.

O objetivo do presente trabalho é comparar diferentes formas de apresentação de alternativas projetuais por meio da Realidade Aumentada em dois dispositivos de visualização. Essa comparação foi realizada por meio de um workshop para coletar a percepção das pessoas perante o experimento.

\section{Procedimentos Metodológicos}

O contexto de projeto é de Habitação de Interesse Social restrito à solução de implantação de um conjunto habitacional. As alternativas de projeto de implantação foram desenvolvidas numa pesquisa realizada anteriormente por Brigitte (2013) como apresentado na seção Dinâmica Colaborativa Utilizada. O experimento proposto confronta duas soluções projetuais no quesito acessibilidade através de quatro formas de apresentação, sendo três em Realidade Aumentada e uma em papel. As quatro formas de visualização das alternativas de solução foram: (i) em RA com imagens vazadas, (ii) em RA com 
imagens opacas, (iii) em RA com imagens com transparência de $50 \%$, e (iv) em papel. As propostas, i a iii, foram visualizadas através de dois tipos de dispositivos de visualização: RA móvel e RA projetada. A RA móvel foi realizada com tablet, enquanto que a RA projetada foi realizada por projetor multimídia. $O$ esquema do experimento está apresentado na Figura 1 e as referidas imagens aplicadas na RA estão ilustradas na Figura 2.

Foram realizados procedimentos técnicos, elaborado um pré-teste, e os dados foram coletados por meio da execução do workshop. A análise dos dados foi feita mediante observação direta do pesquisador e de questionamentos realizados durante o workshop. Os questionamentos foram realizados: (1) após a apresentação das alternativas nas visualizações (i), (ii) e (ii) perguntando-se entre estas qual permitiu melhor comparação das soluções; (2) em seguida perguntou-se a preferência e justificativa entre dispositivos (RA móvel x RA projetada) e (3) finalmente indagou-se sobre a preferência entre confrontação das alternativas de projeto em RA ou no papel.

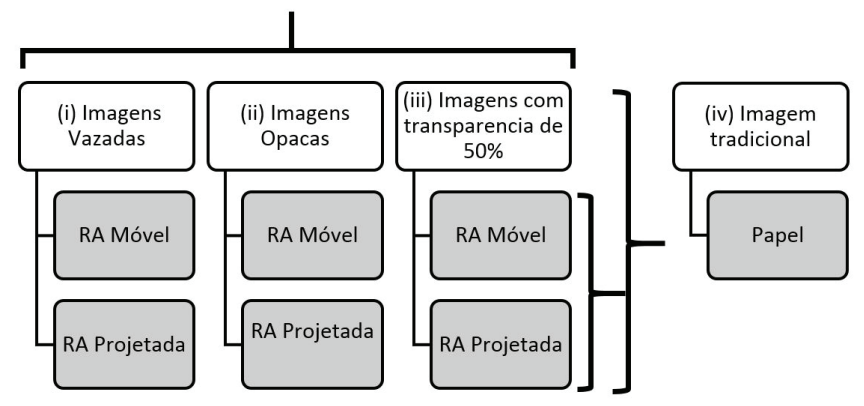

Figura 1: Esquema do experimento. Fonte: Os autores.

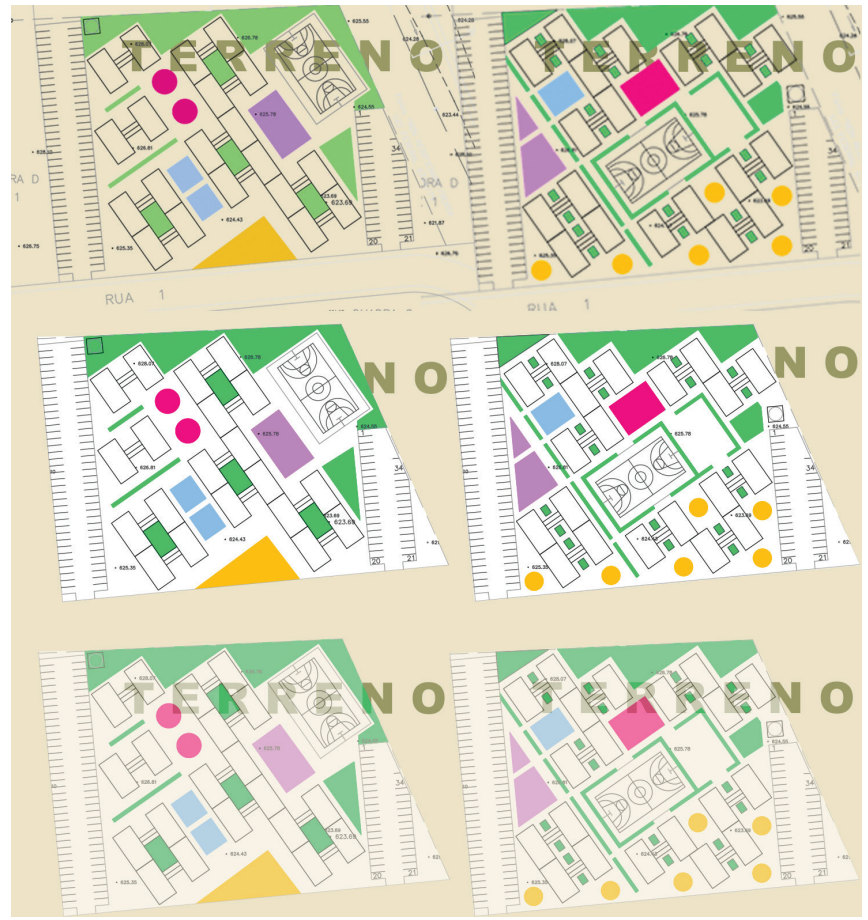

Figura 2: Soluções projetuais do quesito acessibilidade: a) Imagens vazadas, b) Imagens opacas, c) Imagens com 50\% de transparência. Fonte: Os autores.
Vale ressaltar que esse trabalho faz parte do Projeto de Pesquisa em Tecnologias da Informação e Comunicação Aplicadas à Construção de Habitações de Interesse Social (Projeto TIC-HIS), financiado pela FINEP (Financiadora de Estudos e Projetos), e corresponde à meta 30 do Subprojeto 03 - Concepção e Projeto Digital. O referido projeto tem como objetivo estudar e gerar um procedimento para criação de uma interface baseada nas TIC's para a projetação e visualização de edificação em ambientes colaborativos.

\section{Dinâmica Colaborativa Utilizada}

Visando uma aproximação da visualização de alternativas de projetos de arquitetura com a tecnologia de RA, planejou-se implementar a dinâmica desenvolvida por Brigitte (2013) em sua dissertação intitulada "Integração de Desempenho na Avaliação de Projeto: modelo de informação e simulação computacional na etapa de concepção", realizada na Universidade Estadual de Campinas (UNICAMP). O estudo de Brigitte (2013) apresenta uma dinâmica colaborativa que envolve um processo de tomada de decisão por meio da comparação de alternativas de soluções projetuais, na fase de concepção. Essa dinâmica visa o apoio a tomada de decisão e estímulo criativo para a colaboração em diferentes áreas tendo como finalidade uma melhoria na qualidade final do projeto. O processo colaborativo é parte integrante do processo projetual e principalmente nas áreas de AEC, a atividade colaborativa anda em conjunto com tomadas de decisões durante quase todo o processo (BRIGITTE, 2013).

A dinâmica de Brigitte (2013) estimula a colaboração entre diferentes especialidades para a proposição da implantação de um conjunto habitacional. A colaboração é estimulada por meio de um jogo, onde os participantes devem obter a melhor implantação para 10 edificações, 1 quadra e 1 reservatório, de acordo com especialidades específicas. Seis especialidades foram abordadas: conforto acústico, conforto térmico, conforto luminoso, acessibilidade, consumo de água e consumo de materiais.

O desenvolvimento da implantação se dá em três etapas. Na etapa 1 soluções projetuais são obtidas para cada especialidade. Na etapa 2 duas especialidades são confrontadas. $\mathrm{Na}$ etapa 3 sequências de confrontamento entre especialidades são realizadas. A Figura 3 apresenta o esquema da dinâmica proposta por Brigitte (2013).

Este experimento replica parcialmente a etapa 1B da dinâmica de Brigitte (2013), onde cada participante desenvolve uma solução de implantação a partir de decisões individuais (etapa 1A na Figura 3) e reavalia esta proposta comparando-a a simulações de desempenho relacionado a sua especialidade (etapa 1B na Figura 3). Entretanto, neste estudo o confrontamento se dá sempre entre duas soluções, não entre três como originalmente praticado por Brigitte (2013). 


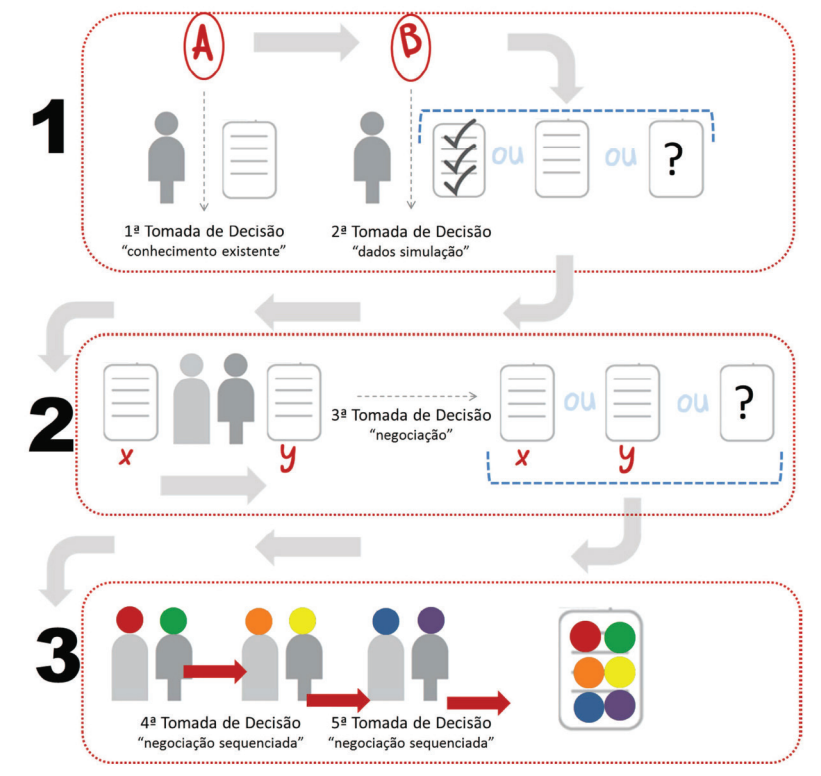

Figura 3: Dinâmica do Jogo. Fonte: Brigitte (2013).

\section{Procedimentos Técnicos Aplicados}

Em uma aplicação de RA são necessários conhecimentos em programas gráficos. $\mathrm{O}$ uso de programas gráficos precede a implementação da aplicação de RA visando a criação do objeto virtual. Os objetos virtuais podem ser: imagens, modelo 2D ou 3D, texto, som, animação ou vídeo. O programa gráfico utilizado para o experimento proposto foi o Adobe Photoshop, da empresa Adobe. Neste programa foram criadas as imagens dos marcadores e as imagens das soluções projetuais, conforme detalhado na seção Procedimentos Metodológicos e ilustrado na Figura 2. Na técnica com marcadores, "o objeto a ser rastreado é um padrão artificial que a princípio não faz parte do cenário e possui formas geométricas que facilitam a identificação do mesmo no ambiente" (SILVA et al., 2012). Após a criação das imagens seguiu para implementação da aplicação de RA.

Os programas de RA utilizados foram o Metaio Creator $6 \mathrm{e}$ o Junaio, ambos da Metaio, empresa recentemente adquirida pela Apple. O primeiro passo foi a inserção das imagens dos marcadores no Metaio Creator. Ao habilitar um marcador, o programa pontua a qualidade do registro de 0 a 3 . Como recomendação para se obter uma boa qualidade do marcador, deve-secriarimagensassimétricasecomarestas bem definidas. Os marcadores criados para esta aplicação obtiveram o nível 2, que corresponde a uma boa qualidade. Para o experimento, a estratégia elaborada foi criar dois marcadores com suas respectivas imagens (a serem visualizadas em RA) deslocadas do centro (uma à esquerda e outra à direita do marcador). Esta estratégia foi adotada para viabilizar a visualização simultânea das imagens (em RA) sobrepostas no terreno, conforme Figura 4. Essas imagens sofreram ajustes de escala para se adequar à escala do terreno impresso.

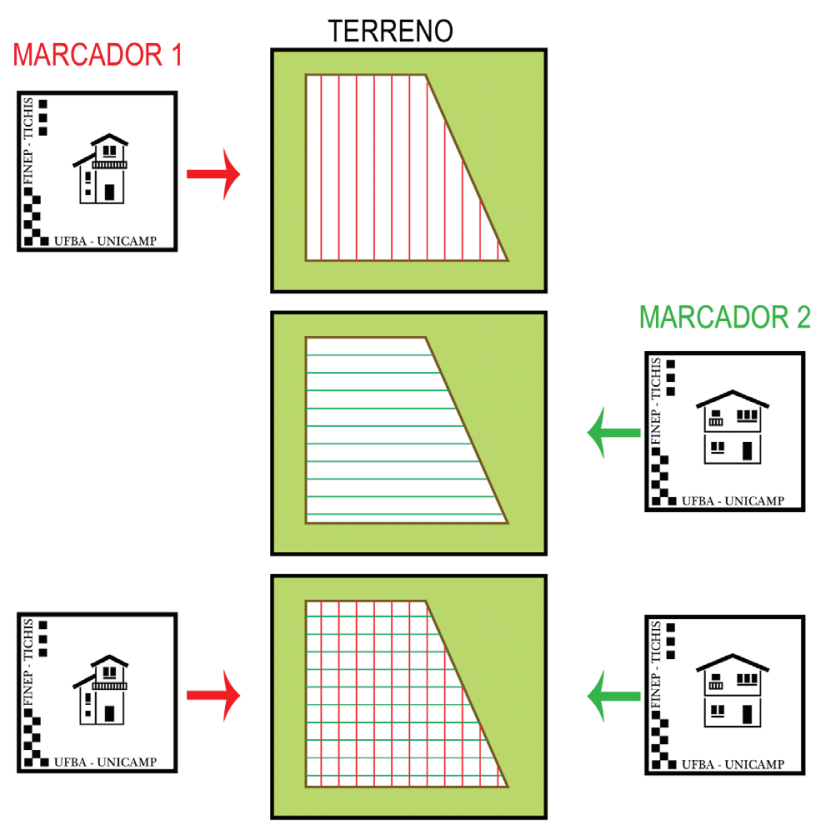

Figura 4: Estratégia para sobreposição de imagens em RA: a) Imagem deslocada a direita do marcador, b) Imagem deslocada à esquerda do marcador, c) Sobreposição de Imagens. Fonte: Os autores.

Para cada tipo de imagem das soluções de implantação no Metaio Creator foi criado um canal de visualização no aplicativo Junaio (http://www.junaio.com/). O canal criado possibilita a visualização da aplicação em RA a partir da leitura de um QR-Code, cada tipo de imagem tem o seu respectivo QR-Code. A Figura 5 apresenta o esquema de associação do QR-Code à uma aplicação e os 3 QR-Codes elaborados para cada tipo de solução de implantação desenvolvida em RA. Isto é necessário para a visualização através de dispositivos móveis como smartphones e tablets.

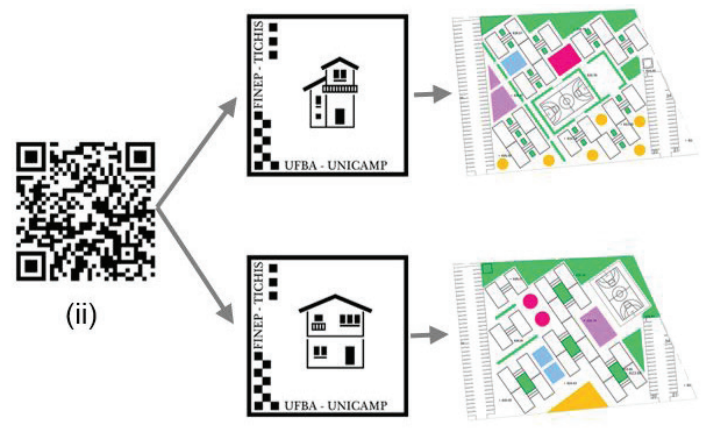

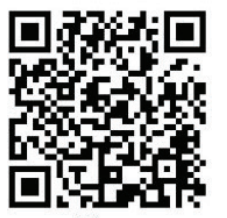

(i)

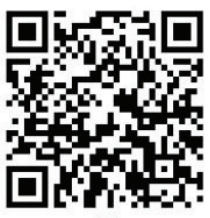

(ii)

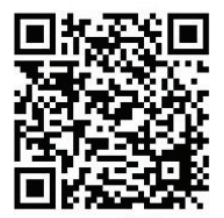

(iii)
Figura 5: Esquema de associação de QR-Code à uma aplicação e QRCodes utilizados: (i) imagens vazadas, (ii) imagens opacas, (iii) imagens com transparência de 50\%. Fonte: Os autores. 
É possível visualizar as imagens em RA do presente trabalho instalando o aplicativo Junaio e posicionando a câmera do dispositivo para cada QR-Code acima e acionando o botão "Scan", em seguida deve-se direcionar a câmera para os marcadores. É necessária a conexão via internet.

\section{Resultados e discussão}

Para a apresentação do experimento foi realizado um workshop no Laboratório de Arquitetura, Metodologia de Projeto e Automações (LAMPA), da Faculdade de Engenharia Civil, Arquitetura e Urbanismo da UNICAMP, em novembro de 2014. Cada tipo de solução foi apresentada simulando uma discussão projetual. A apresentação foi realizada por meio dos dispositivos tablet e projetor multimídia. Após a apresentação das alternativas por meio da RA foram feitos os seguintes questionamentos: (1) qual formato permitiu melhor comparação das soluções, (2) qual a preferência e justificativa entre dispositivos (RA móvel x RA projetada) e finalmente (3) qual a preferência entre a confrontação das alternativas de projeto: RA ou no papel.

A análise dos dados apontou as seguintes considerações, por parte do público presente (cinco participantes, alunos de pós-graduação ou professores da instituição, arquitetos ou engenheiros): para o primeiro questionamento, a proposta com imagens com transparência de 50\% foi indicada com unanimidade a melhor por ser considerada a menos invasiva e mais incorporável ao terreno, pois possibilitou ao usuário visualizar o terreno através da imagem (se houvessem demolições a serem feitas ou vegetação a serem mantidas, por exemplo, estes elementos existentes no terreno poderiam ser vistos). A proposta com imagens vazadas foi considerada a menos indicada e confusa, pois o contorno das edificações se confundia com o terreno gerando um ruído na sua interpretação. Por fim, outras propostas foram sugeridas, como por exemplo a inserção das edificações em RA com apenas as edificações modeladas em 3D.

Com relação aos dispositivos de visualização, RA Projetada x RA Móvel, a RA projetada foi considerada a melhor para este estudo, pois possibilita um maior campo de visão e permite uma maior interação entre pessoas, contribuindo para a discussão entre os participantes. Já ao dispositivo móvel (tablet) foi atribuído uma melhor qualidade de imagem, porém seu manuseio fica restrito a uma pessoa por vez. A figura 6 apresenta as duas formas de visualização realizadas no workshop: RA projetada x RA móvel. E por fim, ao confrontar as alternativas de projeto em RA e a apresentação tradicional em papel, a realidade aumentada foi considerada como mais uma opção viável para discussão projetual em arquitetura.

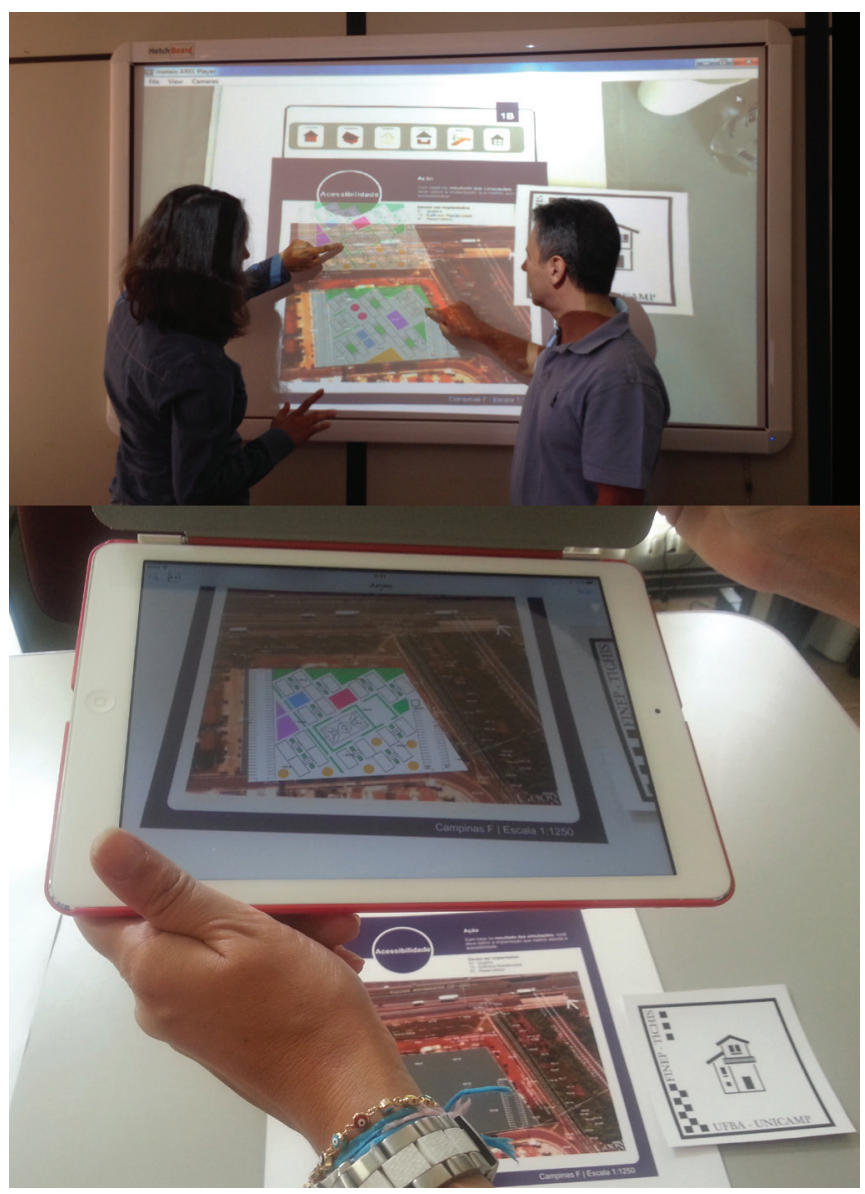

Figura 6: Propostas de visualização do estudo: RA Projetada e RA Móvel. Fonte: Os autores.

\section{Considerações Finais}

O estudo apresentado demonstrou um experimento a ser incorporado na visualização de alternativas projetuais em arquitetura por meio da RA. A utilização da estratégia de visualização de imagens simultâneas em RA contribuiu para uma melhoria no entendimento de propostas projetuais, com interação entre os participantes e auxílio na tomada de decisões. A utilização da RA móvel x RA projetada comparou dispositivos de visualização, sendo a RA projetada a melhor avaliada para a dinâmica de colaboração. Portanto, o uso da RA na visualização em soluções de projeto de arquitetura mostra-se mais adequado com o uso de projetor juntamente com a sobreposição de imagens e múltiplos marcadores. A contribuição deste trabalho está na identificação da forma mais apropriada (imagem com transparência) para análise comparativa de soluções projetuais com RA, na identificação do dispositivo de visualização em RA para apoio à discussão 
(RA projetada) e na forma inovadora de emprego de RA (marcadores com imagens deslocadas). Vale ressaltar que o número de participantes no estudo foi reduzido, sendo os resultados indicativos. Sugere-se novos estudos envolvendo a RA no processo de visualização de soluções projetuais e seu desenvolvimento em outras etapas da atividade projetual. A comparação entre outros tipos de dispositivos, como exemplo óculos de RA, também são nichos de pesquisa nessa área.

\section{Agradecimentos}

Agradecemos a bolsa de estudos, inicialmente pela FINEP e posteriormente pela CAPES, concedida a uma das autoras.

\section{Referencias}

AGUIAR, M. O. Realidade Aumentada: Aplicação em Projetos de Arquitetura e Urbanismo. Florianópolis: UFSC, 2012. 183p. Dissertação (Mestrado em Arquitetura e Urbanismo). Programa de Pós-Graduação em Arquitetura e Urbanismo, Centro Tecnológico, Universidade Federal de Santa Catarina, Florianópolis, 2012.

AZUMA, R. T. A Survey of Augmented Reality. Presence: Teleoperators and Virtual Environments, Columbus, v. 6, n. 4, p. 355-385, 1997.

BRIGITTE, Giovanna T. N. Integração de Desempenho na Avaliação de Projeto: modelo de informação e simulação computacional na etapa de concepção. 2013. 255p. Dissertação (Mestrado em Arquitetura, Tecnologia e Cidade) - Faculdade de Engenharia Civil, Arquitetura e Urbanismo, Universidade
Estadual de Campinas, Campinas, 2013.

CUPERSCHMID, A. R. M. Realidade Aumentada no processo de projeto participativo arquitetônico: desenvolvimento de sistema e diretrizes para utilização. 2014. Tese (Doutorado em Arquitetura e Urbanismo) - Faculdade de Engenharia Civil, Arquitetura e Urbanismo, Universidade Estadual de Campinas, Campinas.

MOREIRA, L. C. S. Expressão Gráfica através da Realidade Aumentada e BIM: uma experiência de visualização. In: SIMPÓSIO NACIONAL DE GEOMETRIA DESCRITIVA E DESENHO TÉCNICO, 21; INTERNATIONAL CONFERENCE ON GRAPHICS ENGINEERING FOR ARTS AND DESIGN, 10., 2013, Florianópolis. Anais... Florianópolis: 2013.

FREITAS, M. R. de; RUSCHEL, R. C. Validação de aplicativo comercial visando à incorporação da realidade aumentada a um modelo de avaliação pós-ocupação. Ambiente Construído, Porto Alegre, v. 15, n. 2, p. 97-112, abr./jun. 2015.

SHIRAZI, A.; BEHZADAN, A. H. Design and Assessment of a Mobile Augmented Reality-Based Information Delivery Tool for Construction and Civil Engineering Curriculum. Journal of Professional Issues in Engineering Education \& Practice, ASCE, 2014.

SILVA, Daliton etal.Qualcomm Vuforia: Realidade Aumentada para Dispositivos IOS com Qualidade e Desempenho. In: XIV SIMPÓSIO DE REALIDADE VIRTUAL E AUMENTADA, 2012, Niterói. Anais... Niterói: SBC, 2012. p. 35-64.

XIANGYU et al. Augmented Reality in built environment: Classification and implications for future research. Automation in Construction, v.32, 2013. p. 1-13 